OPEN ACCESS

Edited by:

Ming Li,

Zhejiang University, China

Reviewed by:

Yu Sun,

Our Lady of the Lake University, United States

Zhenxia Liu,

Linköping University, Sweden

*Correspondence:

Han Gao

1061760802@qq.com

Specialty section: This article was submitted to Interdisciplinary Physics, a section of the journal

Frontiers in Physics

Received: 14 October 2021 Accepted: 05 November 2021

Published: 14 January 2022

Citation:

Gao H, Guo R, Jin Y and Yan L (2022) Large Time Behavior on the Linear SelfInteracting Diffusion Driven by SubFractional Brownian Motion With Hurst Index Large Than 0.5 I: Self-

Repelling Case.

Front. Phys. 9:795210.

doi: 10.3389/fphy.2021.795210

\section{Large Time Behavior on the Linear Self-Interacting Diffusion Driven by Sub-Fractional Brownian Motion With Hurst Index Large Than 0.5 I: Self-Repelling Case}

\author{
Han Gao ${ }^{1 *}$, Rui Guo ${ }^{2}$, Yang Jin $^{3}$ and Litan Yan ${ }^{3}$ \\ ${ }^{1}$ College of Fashion and Art Design, Donghua University, Shanghai, China, ${ }^{2}$ College of Information Science and Technology, \\ Donghua University, Shanghai, China, ${ }^{3}$ Department of Statistics, College of Science, Donghua University, Shanghai, China
}

Let $S^{H}$ be a sub-fractional Brownian motion with index $\frac{1}{2}<H<1$. In this paper, we consider the linear self-interacting diffusion driven by $S^{H}$, which is the solution to the equation

$$
d X_{t}^{H}=d S_{t}^{H}-\theta\left(\int_{0}^{t}\left(X_{t}^{H}-X_{s}^{H}\right) d s\right) d t+v d t, \quad X_{0}^{H}=0,
$$

where $\theta<0$ and $v \in \mathbb{R}$ are two parameters. Such process $X^{H}$ is called self-repelling and it is an analogue of the linear self-attracting diffusion [Cranston and Le Jan, Math. Ann. 303 (1995), 87-93]. Our main aim is to study the large time behaviors. We show the solution $X^{H}$ diverges to infinity, as $t$ tends to infinity, and obtain the speed at which the process $X^{H}$ diverges to infinity as $t$ tends to infinity.

Keywords: the self-repelling diffusion, asymptotic distribution, convergence, sub-fractional Brownian motion, stochastic integral

\section{INTRODUCTION}

In 1995, Cranston and Le Jan [1] introduced a linear self-attracting diffusion

$$
X_{t}=B_{t}-\theta \int_{0}^{t} \int_{0}^{s}\left(X_{s}-X_{u}\right) d u d s+\nu t, \quad t \geq 0
$$

with $\theta>0$ and $X_{0}=0$, where $B$ is a 1-dimensional standard Brownian motion. They showed that the process $X_{t}$ converges in $L^{2}$ and almost surely, as $t$ tends infinity. This is a special case of path dependent stochastic differential equations. Such path dependent stochastic differential equation was first developed by Durrett and Rogers [2] introduced in 1992 as a model for the shape of a growing polymer (Brownian polymer) as follows

$$
X_{t}=X_{0}+B_{t}+\int_{0}^{t} \int_{0}^{s} f\left(X_{s}-X_{u}\right) d u d s,
$$

where $B$ is a $d$-dimensional standard Brownian motion and $f$ is Lipschitz continuous. $X_{t}$ corresponds to the location of the end of the polymer at time $t$. Under some conditions, they established asymptotic behavior of the solution of stochastic differential equation and gave some conjectures and questions. The model is a continuous analogue of the notion of edge (resp. vertex) self-interacting random walk. If $f(x)=g(x) x /\|x\|$ and $g(x) \geq 0, X_{t}$ is a continuous analogue of a process introduced by 
Diaconis and studied by Pemantle [3]. Let $\mathscr{L}^{X}(t, x)$ be the local time of the solution process $X$. Then, we have

$$
X_{t}=X_{0}+B_{t}+\int_{0}^{t} d s \int_{\mathbb{R}} f(-x) \mathscr{L}^{X}\left(s, X_{s}+x\right) d x
$$

for all $t \geq 0$. This formulation makes it clear how the process $X$ interacts with its own occupation density. We may call this solution a Brownian motion interacting with its own passed trajectory, i.e., a self-interacting motion. In general, the Eq. 1.2 defines a self-interacting diffusion without any assumption on $f$. If

$$
x \cdot f(x) \geq 0 \quad(x \cdot f(x) \leq 0)
$$

for all $x \in \mathbb{R}^{d}$, we call it self-repelling (resp. self-attracting). In 2002, Benaïm et al [4] also introduced a self-interacting diffusion with dependence on the (convolved) empirical measure. A great difference between these diffusions and Brownian polymers is that the drift term is divided by $t$. It is noteworthy that the interaction potential is attractive enough to compare the diffusion (a bit modified) to an Ornstein-Uhlenbeck process, in many case of $f$, which points out an access to its asymptotic behavior. More works can be found in Benaïm et al. [5], Cranston and Mountford [6], Gauthier [7], Herrmann and Roynette [8], Herrmann and Scheutzow [9], Mountford and Tarr [10], Shen et al [11], Sun and Yan [12] and the references therein.

On the other hand, starting from the application of fractional Brownian motion in polymer modeling, Yan et al [13] considered an analogue of the linear self-interacting diffusion:

$$
X_{t}^{H}=B_{t}^{H}-\theta \int_{0}^{t} \int_{0}^{s}\left(X_{s}^{H}-X_{u}^{H}\right) d u d s+v t, \quad t \geq 0
$$

with $\theta \neq 0$ and $X_{0}^{H}=0$, where $B^{H}$ is a fractional Brownian motion (fBm, in short) with Hurst parameter $\frac{1}{2} \leq H<1$. The solution of (1.3) is a Gaussian process. When $\theta>0$, Yan et al [13] showed that the solution $X^{H}$ of (1.3) converges in $L^{2}$ and almost surely, to the random variable

$$
X_{\infty}^{H}=\int_{0}^{\infty} h_{\theta}(s) d B_{s}^{H}+v \int_{0}^{\infty} h_{\theta}(s) d s
$$

where the function is defined ar follows

$$
h_{\theta}(s)=1-\theta s e^{\frac{1}{2} \theta s^{2}} \int_{s}^{\infty} e^{-\frac{1}{2} \theta u^{2}} d u, \quad s \geq 0
$$

with $\theta>0$. Recently, Sun and Yan [14] considered the related parameter estimations with $\theta>0$ and $\frac{1}{2} \leq H<1$, and Gan and Yan [15] considered the parameter estimations with $\theta<0$ and $\frac{1}{2} \leq H<1$.

Motivated by these results, as a natural extension one can consider the following stochastic differential equation:

$$
X_{t}=G_{t}-\theta \int_{0}^{t} \int_{0}^{s}\left(X_{s}-X_{u}\right) d u d s+v t, \quad t \geq 0
$$

with $\theta>0$ and $X_{0}=0$, where $G=\left\{G_{t}, t \geq 0\right\}$ is a Gaussian process with some suitable conditions which includes fractional Brownian motion and some related processes. However, for a (general) abstract Gaussian process it is difficult to find some interesting fine estimates associated with the calculations. So, in this paper we consider the linear self-attracting diffusion driven by a sub-fractional Brownian motion (sub-fBm, in short). We choose this kind of Gaussian process because it is only the generalization of Brownian motion rather than the generalization of fractional Brownian motion. It only has some similar properties of fractional Brownian motion, such as long memory and self similarity, but it has no stationary increment. The so-called sub-fBm with index $H \in(0,1)$ is a mean zero Gaussian process $S^{H}=\left\{S_{t}^{H}, t \geq 0\right\}$ with $S_{0}^{H}=0$ and the covariance

$$
R_{H}(t, s) \equiv E\left[S_{t}^{H} S_{s}^{H}\right]=s^{2 H}+t^{2 H}-\frac{1}{2}\left[(s+t)^{2 H}+|t-s|^{2 H}\right]
$$

for all $s, t \geq 0$. For $H=1 / 2, S^{H}$ coincides with the standard Brownian motion B. $S^{H}$ is neither a semimartingale nor a Markov process unless $H=1 / 2$, so many of the powerful techniques from stochastic analysis are not available when dealing with $S^{H}$. As a Gaussian process, it is possible to construct a stochastic calculus of variations with respect to $S^{H}$ (see, for example, Alós et al [16]). The sub-fBm has properties analogous to those of $\mathrm{fBm}$ and satisfies the following estimates:

$$
\begin{gathered}
{\left[\left(2-2^{2 H-1}\right) \wedge 1\right](t-s)^{2 H} \leq E\left[\left(S_{t}^{H}-S_{s}^{H}\right)^{2}\right]} \\
\leq\left[\left(2-2^{2 H-1}\right) \vee 1\right](t-s)^{2 H} .
\end{gathered}
$$

More works for sub-fBm and related processes can be found in Bojdecki et al. [17-20], Li [21-24], Shen and Yan [25, 26], Sun and Yan [27], Tudor [28-31], Ciprian A. Tudor [32] Yan et al [33-35] and the references therein.

In this present paper, we consider the linear self-interacting diffusion

$$
X_{t}^{H}=S_{t}^{H}-\theta \int_{0}^{t} \int_{0}^{s}\left(X_{s}^{H}-X_{u}^{H}\right) d u d s+v t, \quad t \geq 0
$$

with $\theta<0$ and $X_{0}^{H}=0$, where $S^{H}$ is a sub-fBm with Hurst parameter $\frac{1}{2} \leq H<1$. Our main aim is to show that the solution of (1.7) diverges to infinity and obtain the speed diverging to infinity, as $t$ tends to infinity. The object of this paper is to expound and prove the following statements:

(I) For $\theta<0$ and $\frac{1}{2}<H<1$, the random variable

$$
\xi_{\infty}^{H}=\int_{0}^{\infty} s e^{\frac{1}{2} \theta s^{2}} d S_{s}^{H}
$$

exists as an element in $L^{2}$.

(II) For $\theta<0$ and $\frac{1}{2}<H<1$, as $t \rightarrow \infty$, we have

$$
J_{0}^{H}(t ; \theta, \nu):=t e^{\frac{1}{2} \theta t^{2}} X_{t}^{H} \rightarrow \xi_{\infty}^{H}-\frac{\nu}{\theta}
$$

in $L^{2}$ and almost surely.

(III) For $\theta<0$ and $\frac{1}{2}<H<1$, define the processes $J^{H}(n, \theta, v)=\left\{J_{t}^{H}(n, \theta, v), t \geq 0\right\}, n \geq 1$ by 


$$
\begin{aligned}
J_{n}^{H}(t ; \theta, \nu) & :=\theta t^{2}\left(J_{n-1}^{H}(t ; \theta, \nu)-(2 n-3) ! !\left(\xi_{\infty}^{H}-\frac{\nu}{\theta}\right)\right) \\
n & =1,2, \ldots
\end{aligned}
$$

for all $t \geq 0$, where $(-1) ! !=1$. We then have

$$
J_{n}^{H}(t ; \theta, \nu) \rightarrow(2 n-1) ! !\left(\xi_{\infty}^{H}-\frac{\nu}{\theta}\right)
$$

holds in $L^{2}$ and almost surely for every $n \geq 1$, as $t \rightarrow \infty$.

This paper is organized as follows. In Section 2 we present some preliminaries for sub-fBm and Malliavin calculus. In Section 3, we obtain some lemmas. In Section 4, we prove the main result. In Section 5 we give some numerical results.

\section{PRELIMINARIES}

In this section, we briefly recall the definition and properties of stochastic integral with respect to sub-fBm. We refer to Alós et al [16], Nualart [36], and Tudor [31] for a complete description of stochastic calculus with respect to Gaussian processes. Throughout this paper we assume that $S^{H}=\left\{S_{t}^{H}, t \geq 0\right\}$ denotes a sub-fBm defined on the probability space $(\Omega, \mathcal{F}, P)$ with index $H$. As we pointed out before, the sub-fBm $S^{H}$ is a rather special class of self-similar Gaussian processes such that $S_{0}^{H}=0$, $E\left[S_{t}^{H}\right]=0$ and

$$
R^{H}(t, s):=E\left[S_{t}^{H} S_{s}^{H}\right]=s^{2 H}+t^{2 H}-\frac{1}{2}\left[(s+t)^{2 H}+|t-s|^{2 H}\right]
$$

for all $s, t \geq 0$. For $H=1 / 2, S^{H}$ coincides with the standard Brownian motion B. $S^{H}$ is neither a semimartingale nor a Markov process unless $H=1 / 2$, so many of the powerful techniques from stochastic analysis are not available when dealing with $S^{H}$. As a Gaussian process, it is possible to construct a stochastic calculus of variations with respect to $S^{H}$. The sub-fBm appeared in Bojdecki et al [17] in a limit of occupation time fluctuations of a system of independent particles moving in $\mathbb{R}^{d}$ according a symmetric $\alpha$-stable Lévy process, and it also appears in Bojdecki et al [18] in a high-density limit of occupation time fluctuations of the above mentioned particle system, where the initial Poisson configuration has finite intensity measure.

The estimate (1.6) and normality imply that the sub-fBm $t \mapsto S_{t}^{H}$ admits almost surely a bounded $\frac{1}{H-9}$-variation on any finite interval for any sufficiently small $\vartheta \in(0, H)$. That is, the paths of $t \mapsto S_{t}^{H}$ admits a bounded $p_{H}$-variation on any finite interval with $p_{H}>\frac{1}{H}$. As an immediate result, one can define the Young integral of a process $u=\left\{u_{t}, t \geq 0\right\}$ with respect to sub$\mathrm{fBm} B^{a, b}$

$$
\int_{0}^{t} u_{s} d S_{s}^{H}
$$

as the limit in probability of a Riemann sum. Clearly, the integral is well-defined and

$$
u_{t} S_{t}^{H}=\int_{0}^{t} u_{s} d S_{s}^{H}+\int_{0}^{t} S_{s}^{H} d u_{s}
$$

for all $t \geq 0$, provided $u$ is of bounded $q_{H}$-variation on any finite interval with $q_{H}>1$ and $\frac{1}{p_{H}}+\frac{1}{q_{H}}>1$ (see, for examples, Bertoin [37] and FöIllmer [38]).

Let $\mathcal{H}$ be the completion of the linear space $\mathcal{E}$ generated by the indicator functions $1_{[0, t]}, t \in[0, T]$ with respect to the inner product

$$
\left\langle 1_{[0, s]}, 1_{[0, t]}\right\rangle_{\mathcal{H}}=R^{H}(t, s)
$$

for $s, t \in[0, T]$. When $\frac{1}{2}<H<1$, we can show that

$$
\begin{aligned}
\|\varphi\|_{\mathcal{H}}^{2} & =\int_{0}^{T} \int_{0}^{T} \varphi(t) \varphi(s) \frac{\partial^{2}}{\partial t \partial s} R^{H}(t, s) d s d t \\
& =\int_{0}^{T} \int_{0}^{T} \varphi(t) \varphi(s) \psi_{H}(t, s) d s d t, \quad \forall \varphi \in \mathcal{H},
\end{aligned}
$$

where

$$
\psi_{H}(t, s)=\frac{\partial^{2}}{\partial t \partial s} R^{a, b}(t, s)=H(2 H-1)\left(|t-s|^{2 H-2}-|t+s|^{2 H-2}\right)
$$

for $s, t \in[0, T]$. Define the linear mapping $\mathcal{E} \ni \varphi \mapsto S^{H}(\varphi)$ by

$$
1_{[0, t]} \mapsto S^{H}\left(1_{[0, t]}\right)=\int_{0}^{T} 1_{[0, t]}(s) d S_{s}^{H} \equiv S_{t}^{H}
$$

for all $t \in[0, T]$ and it can be continuously extended to $\mathcal{H}$ and we call the mapping $\Phi$ is called the Wiener integral with respect to $S^{H}$, denoted by

$$
S^{H}(\varphi)=\int_{0}^{T} \varphi(s) d S_{s}^{H}
$$

and

$$
\|\varphi\|_{\mathcal{H}}^{2}=E\left(\int_{0}^{T} \varphi(s) d S_{s}^{H}\right)^{2}
$$

for any $\varphi \in \mathcal{H}$.

For simplicity, in this paper we assume that $\frac{1}{2}<H<1$. Thus, if for every $T>0$, the integral

$$
\int_{0}^{T} \varphi(s) d S_{s}^{H}
$$

exists in $L^{2}$ and

$$
\int_{0}^{\infty} \int_{0}^{\infty} \varphi(t) \varphi(s) \psi_{H}(t, s) d s d t<\infty,
$$

we can define the integral

$$
\int_{0}^{\infty} \varphi(s) d S_{s}^{H}
$$

and

$$
E\left(\int_{0}^{\infty} \varphi(s) d S_{s}^{H}\right)^{2}=\int_{0}^{\infty} \int_{0}^{\infty} \varphi(t) \varphi(s) \psi_{H}(t, s) d s d t
$$

Denote by $\mathcal{S}$ the set of smooth functionals of the form

$$
F=f\left(S^{H}\left(\varphi_{1}\right), S^{H}\left(\varphi_{2}\right), \ldots, S^{H}\left(\varphi_{n}\right)\right),
$$

where $f \in C_{b}^{\infty}\left(\mathbb{R}^{n}\right)$ and $\varphi_{i} \in \mathcal{H}$. The Malliavin derivative $D$ of a functional $F$ as above is given by 


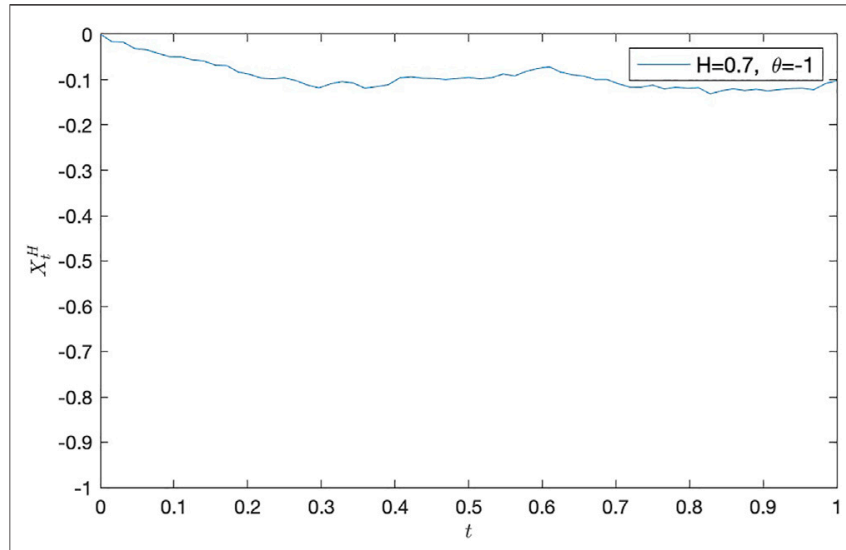

FIGURE 1 | A path of $X^{H}$ with $\theta=-1$ and $H=0.7$.

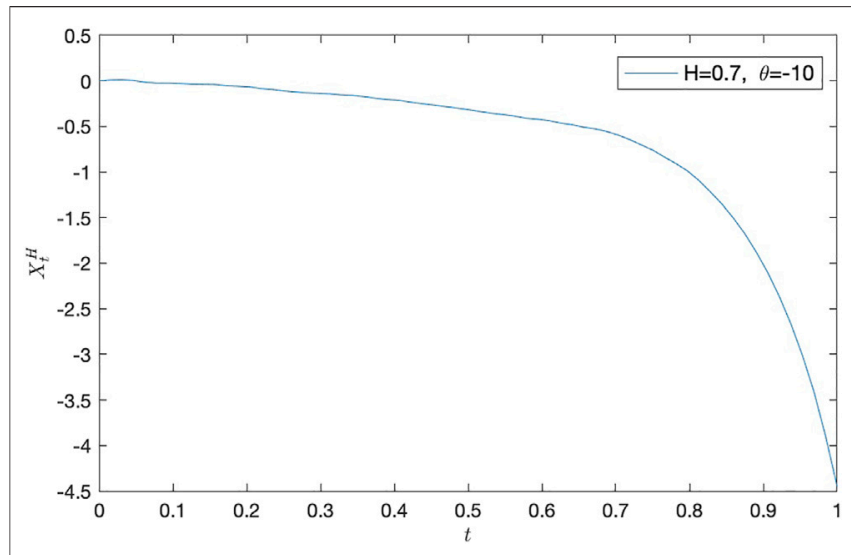

FIGURE 2 | A path of $X^{H}$ with $\theta=-10$ and $H=0.7$.

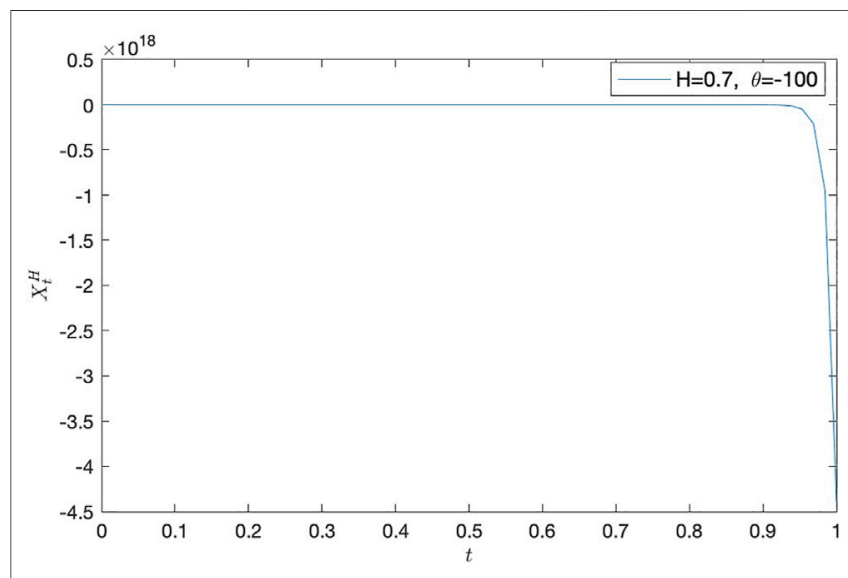

FIGURE $3 \mid$ A path of $X^{H}$ with $\theta=-100$ and $H=0.7$.

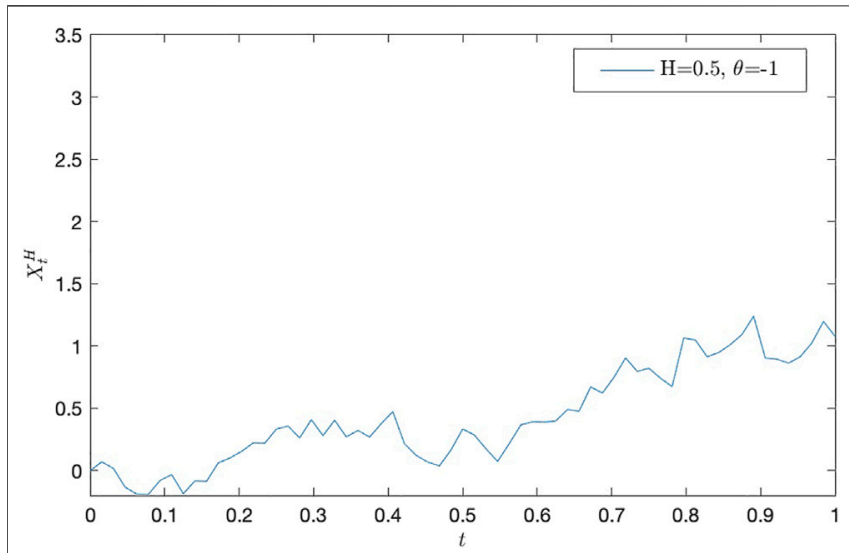

FIGURE $4 \mid A$ path of $X^{H}$ with $\theta=-1$ and $H=0.5$.

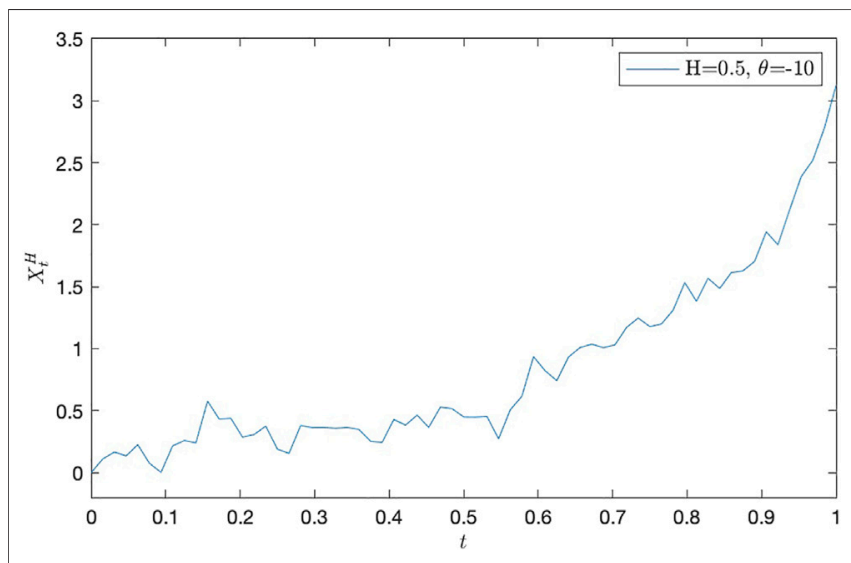

FIGURE 5 | A path of $X^{H}$ with $\theta=-10$ and $H=0.5$.

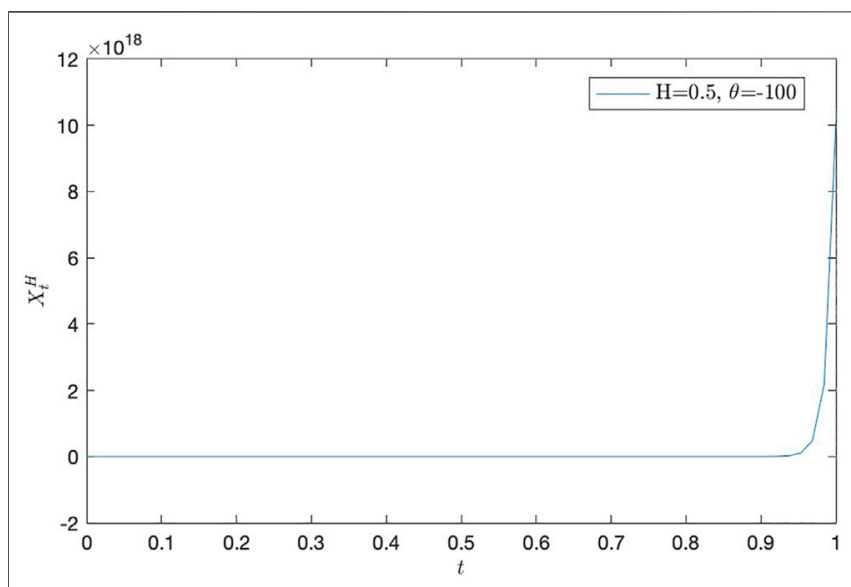

FIGURE 6 | A path of $X^{H}$ with $\theta=-100$ and $H=0.5$. 
TABLE 1 | The data of $X_{t}^{H}$ with $\theta=-1$ and $H=0.7$.

\begin{tabular}{lccccc}
\hline $\boldsymbol{t}$ & $\mathbf{X}_{\mathbf{t}}^{\mathbf{H}}$ & $\boldsymbol{T}$ & $\mathbf{X}_{\mathbf{t}}^{\mathbf{H}}$ & $\boldsymbol{t}$ & $\mathbf{X}_{\mathbf{t}}^{\mathbf{H}}$ \\
\hline 0.0000 & 0.0000 & 0.3438 & -0.1077 & 0.6875 & -0.0995 \\
0.0156 & -0.0167 & 0.3594 & -0.1190 & 0.7031 & -0.1091 \\
0.0313 & -0.0178 & 0.3750 & -0.1153 & 0.7188 & -0.1163 \\
0.0469 & -0.0320 & 0.3906 & -0.1116 & 0.7344 & -0.1165 \\
0.0625 & -0.0338 & 0.4063 & -0.0965 & 0.7500 & -0.1122 \\
0.0781 & -0.0420 & 0.4219 & -0.0937 & 0.7656 & -0.1205 \\
0.0938 & -0.0492 & 0.4375 & -0.0971 & 0.7813 & -0.1170 \\
0.1094 & -0.0496 & 0.4531 & -0.0974 & 0.7969 & -0.1192 \\
0.1250 & -0.0564 & 0.4688 & -0.0997 & 0.8125 & -0.1180 \\
0.1406 & -0.0590 & 0.4844 & -0.0976 & 0.8281 & -0.1316 \\
0.1563 & -0.0682 & 0.5000 & -0.0956 & 0.8438 & -0.1245 \\
0.1719 & -0.0692 & 0.5156 & -0.0983 & 0.8594 & -0.1202 \\
0.1875 & -0.0834 & 0.5313 & -0.0959 & 0.8750 & -0.1241 \\
0.2031 & -0.0886 & 0.5469 & -0.0877 & 0.8906 & -0.1212 \\
0.2188 & -0.0969 & 0.5625 & -0.0919 & 0.9063 & -0.1250 \\
0.2344 & -0.0983 & 0.5781 & -0.0818 & 0.9219 & -0.1219 \\
0.2500 & -0.0961 & 0.5938 & -0.0757 & 0.9375 & -0.1199 \\
0.2656 & -0.1022 & 0.6094 & -0.0717 & 0.9531 & -0.1191 \\
0.2813 & -0.1120 & 0.6250 & -0.0834 & 0.9688 & -0.1223 \\
0.2969 & -0.1182 & 0.6406 & -0.0894 & 0.9844 & -0.1089 \\
0.3125 & -0.1094 & 0.6563 & -0.0923 & 1.0000 & -0.1023 \\
0.3281 & -0.1042 & 0.6719 & -0.0996 & - & - \\
& & & & &
\end{tabular}

\begin{tabular}{lccccc}
\hline \multicolumn{7}{l}{ TABLE 2 } & The data of $X_{t}^{H}$ with $\theta=-10$ and $H=0.7$. & \\
\hline $\boldsymbol{t}$ & $\mathbf{X}_{\mathbf{t}}^{\mathbf{H}}$ & $\boldsymbol{t}$ & $\mathbf{X}_{\mathbf{t}}^{\mathbf{H}}$ & $\boldsymbol{t}$ & $\mathbf{X}_{\mathbf{t}}^{\mathbf{H}}$ \\
\hline 0.0000 & 0.0000 & 0.3438 & -0.1597 & 0.6875 & -0.5552 \\
0.0156 & 0.0087 & 0.3594 & -0.1729 & 0.7031 & -0.5943 \\
0.0313 & 0.0113 & 0.3750 & -0.1912 & 0.7188 & -0.6439 \\
0.0469 & 0.0040 & 0.3906 & -0.2051 & 0.7344 & -0.7019 \\
0.0625 & -0.0153 & 0.4063 & -0.2130 & 0.7500 & -0.7595 \\
0.0781 & -0.0239 & 0.4219 & -0.2342 & 0.7656 & -0.8345 \\
0.0938 & -0.0234 & 0.4375 & -0.2494 & 0.7813 & -0.9066 \\
0.1094 & -0.0279 & 0.4531 & -0.2654 & 0.7969 & -0.9868 \\
0.1250 & -0.0348 & 0.4688 & -0.2820 & 0.8125 & -1.0919 \\
0.1406 & -0.0372 & 0.4844 & -0.2980 & 0.8281 & -1.2177 \\
0.1563 & -0.0395 & 0.5000 & -0.3156 & 0.8438 & -1.3507 \\
0.1719 & -0.0530 & 0.5156 & -0.3363 & 0.8594 & -1.5050 \\
0.1875 & -0.0587 & 0.5313 & -0.3543 & 0.8750 & -1.6776 \\
0.2031 & -0.0648 & 0.5469 & -0.3694 & 0.8906 & -1.8811 \\
0.2188 & -0.0835 & 0.5625 & -0.3865 & 0.9063 & -2.1081 \\
0.2344 & -0.0942 & 0.5781 & -0.4093 & 0.9219 & -2.3699 \\
0.2500 & -0.1100 & 0.5938 & -0.4204 & 0.9375 & -2.6701 \\
0.2656 & -0.1213 & 0.6094 & -0.4368 & 0.9531 & -3.0170 \\
0.2813 & -0.1317 & 0.6250 & -0.4620 & 0.9688 & -3.4144 \\
0.2969 & -0.1365 & 0.6406 & -0.4810 & 0.9844 & -3.8989 \\
0.3125 & -0.1418 & 0.6563 & -0.5086 & 1.0000 & -4.4510 \\
0.3281 & -0.1541 & 0.6719 & -0.5258 & - & - \\
& & & & &
\end{tabular}

$$
D F=\sum_{j=1}^{n} \frac{\partial f}{\partial x_{j}}\left(S^{H}\left(\varphi_{1}\right), S^{H}\left(\varphi_{2}\right), \ldots, S^{H}\left(\varphi_{n}\right)\right) \varphi_{j} .
$$

The derivative operator $D$ is then a closable operator from $L^{2}(\Omega)$ into $L^{2}(\Omega ; \mathcal{H})$. We denote by $\mathbb{D}^{1,2}$ the closure of $\mathcal{S}$ with respect to the norm

$$
\|F\|_{1,2}:=\sqrt{E|F|^{2}+E\|D F\|_{\mathcal{H}}^{2}} .
$$

TABLE 3 | The data of $X_{t}^{H}$ with $\theta=-100$ and $H=0.7$.

\begin{tabular}{lccccc}
\hline $\boldsymbol{t}$ & $\mathbf{X}_{\mathbf{t}}^{\mathbf{H}}$ & $\boldsymbol{t}$ & $\mathbf{X}_{\mathbf{t}}^{\mathbf{H}}$ & $\boldsymbol{t}$ & $\mathbf{X}_{\mathbf{t}}^{\mathbf{H}}$ \\
\hline 0.0000 & 0.0000 & 0.3438 & -1.0056 & 0.6875 & $-2.29 \mathrm{E}+07$ \\
0.0156 & 0.0132 & 0.3594 & -1.6439 & 0.7031 & $-6.63 \mathrm{E}+07$ \\
0.0313 & 0.0093 & 0.3750 & -2.7733 & 0.7188 & $-1.97 \mathrm{E}+08$ \\
0.0469 & 0.0070 & 0.3906 & -4.8028 & 0.7344 & $-5.99 \mathrm{E}+08$ \\
0.0625 & 0.0103 & 0.4063 & -8.5377 & 0.7500 & $-1.87 \mathrm{E}+09$ \\
0.0781 & 0.0116 & 0.4219 & -15.5941 & 0.7656 & $-5.98 \mathrm{E}+09$ \\
0.0938 & 0.0092 & 0.4375 & -29.2598 & 0.7813 & $-1.96 \mathrm{E}+10$ \\
0.1094 & 0.0066 & 0.4531 & -56.3669 & 0.7969 & $-6.59 \mathrm{E}+10$ \\
0.1250 & 0.0081 & 0.4688 & -111.4786 & 0.8125 & $-2.27 \mathrm{E}+11$ \\
0.1406 & 0.0049 & 0.4844 & -226.2866 & 0.8281 & $-8.02 \mathrm{E}+11$ \\
0.1563 & 0.0094 & 0.5000 & -471.3711 & 0.8438 & $-2.91 \mathrm{E}+12$ \\
0.1719 & -0.0029 & 0.5156 & $-1.01 \mathrm{E}+03$ & 0.8594 & $-1.08 \mathrm{E}+13$ \\
0.1875 & -0.0114 & 0.5313 & $-2.21 \mathrm{E}+03$ & 0.8750 & $-4.10 \mathrm{E}+13$ \\
0.2031 & -0.0279 & 0.5469 & $-4.97 \mathrm{E}+03$ & 0.8906 & $-1.60 \mathrm{E}+14$ \\
0.2188 & -0.0484 & 0.5625 & $-1.15 \mathrm{E}+04$ & 0.9063 & $-6.40 \mathrm{E}+14$ \\
0.2344 & -0.0557 & 0.5781 & $-2.72 \mathrm{E}+04$ & 0.9219 & $-2.62 \mathrm{E}+15$ \\
0.2500 & -0.0837 & 0.5938 & $-6.59 \mathrm{E}+04$ & 0.9375 & $-1.10 \mathrm{E}+16$ \\
0.2656 & -0.1240 & 0.6094 & $-1.64 \mathrm{E}+05$ & 0.9531 & $-4.75 \mathrm{E}+16$ \\
0.2813 & -0.1834 & 0.6250 & $-4.19 \mathrm{E}+05$ & 0.9688 & $-2.10 \mathrm{E}+17$ \\
0.2969 & -0.2706 & 0.6406 & $-1.10 \mathrm{E}+06$ & 0.9844 & $-9.48 \mathrm{E}+17$ \\
0.3125 & -0.4085 & 0.6563 & $-2.95 \mathrm{E}+06$ & 1.0000 & $-4.40 \mathrm{E}+18$ \\
0.3281 & -0.6332 & 0.6719 & $-8.12 \mathrm{E}+06$ & - & - \\
& & & & &
\end{tabular}

TABLE 4 | The data of $X_{t}^{H}$ with $\theta=-1$ and $H=0.5$.

\begin{tabular}{lccccc}
$\boldsymbol{t}$ & $\mathbf{X}_{\mathbf{t}}^{\mathbf{H}}$ & $\boldsymbol{t}$ & $\mathbf{X}_{\mathbf{t}}^{\mathbf{H}}$ & $\boldsymbol{t}$ & $\mathbf{X}_{\mathbf{t}}^{\mathbf{H}}$ \\
\hline 0.0000 & 0.0000 & 0.3438 & 0.2713 & 0.6875 & 0.6225 \\
0.0156 & 0.0711 & 0.3594 & 0.3234 & 0.7031 & 0.7483 \\
0.0313 & 0.0168 & 0.3750 & 0.2698 & 0.7188 & 0.9047 \\
0.0469 & -0.1326 & 0.3906 & 0.3765 & 0.7344 & 0.7963 \\
0.0625 & -0.1887 & 0.4063 & 0.4725 & 0.7500 & 0.8221 \\
0.0781 & -0.1911 & 0.4219 & 0.2156 & 0.7656 & 0.7416 \\
0.0938 & -0.0792 & 0.4375 & 0.1224 & 0.7813 & 0.6743 \\
0.1094 & -0.0320 & 0.4531 & 0.0691 & 0.7969 & 1.0655 \\
0.1250 & -0.1853 & 0.4688 & 0.0377 & 0.8125 & 1.0480 \\
0.1406 & -0.0827 & 0.4844 & 0.1668 & 0.8281 & 0.9146 \\
0.1563 & -0.0861 & 0.5000 & 0.3344 & 0.8438 & 0.9478 \\
0.1719 & 0.0616 & 0.5156 & 0.2866 & 0.8594 & 1.0125 \\
0.1875 & 0.1014 & 0.5313 & 0.1759 & 0.8750 & 1.0931 \\
0.2031 & 0.1542 & 0.5469 & 0.0739 & 0.8906 & 1.2403 \\
0.2188 & 0.2224 & 0.5625 & 0.2168 & 0.9063 & 0.9036 \\
0.2344 & 0.2205 & 0.5781 & 0.3676 & 0.9219 & 0.8949 \\
0.2500 & 0.3345 & 0.5938 & 0.3904 & 0.9375 & 0.8626 \\
0.2656 & 0.3581 & 0.6094 & 0.3878 & 0.9531 & 0.9140 \\
0.2813 & 0.2635 & 0.6250 & 0.3985 & 0.9688 & 1.0247 \\
0.2969 & 0.4084 & 0.6406 & 0.4900 & 0.9844 & 1.1976 \\
0.3125 & 0.2820 & 0.6563 & 0.4769 & 1.0000 & 1.0780 \\
0.3281 & 0.4043 & 0.6719 & 0.6713 & - & - \\
& & & & &
\end{tabular}

The divergence integral $\delta$ is the adjoint of derivative operator $D^{H}$. That is, we say that a random variable $u$ in $L^{2}(\Omega ; \mathcal{H})$ belongs to the domain of the divergence operator $\delta$, denoted by $\operatorname{Dom}\left(\delta^{S}\right)$, if

$$
E\left|\langle D F, u\rangle_{\mathcal{H}}\right| \leq c\|F\|_{L^{2}(\Omega)}
$$

for every $F \in \mathbb{D}^{1,2}$, where $c$ is a constant depending only on $u$. In this case $\delta(u)$ is defined by the duality relationship

$$
E[F \delta(u)]=E\langle D F, u\rangle_{\mathcal{H}}
$$


TABLE 5 | The data of $X_{t}^{H}$ with $\theta=-10$ and $H=0.5$.

\begin{tabular}{lccccc}
\hline $\boldsymbol{t}$ & $\mathbf{X}_{\mathbf{t}}^{\mathbf{H}}$ & $\boldsymbol{t}$ & $\mathbf{X}_{\mathbf{t}}^{\mathbf{H}}$ & $\boldsymbol{t}$ & $\mathbf{X}_{\mathbf{t}}^{\mathbf{H}}$ \\
\hline 0.0000 & 0.0000 & 0.3438 & 0.3643 & 0.6875 & 1.0084 \\
0.0156 & 0.1112 & 0.3594 & 0.3489 & 0.7031 & 1.0312 \\
0.0313 & 0.1668 & 0.3750 & 0.2532 & 0.7188 & 1.1722 \\
0.0469 & 0.1353 & 0.3906 & 0.2453 & 0.7344 & 1.2474 \\
0.0625 & 0.2259 & 0.4063 & 0.4297 & 0.7500 & 1.1783 \\
0.0781 & 0.0764 & 0.4219 & 0.3837 & 0.7656 & 1.1997 \\
0.0938 & 0.0025 & 0.4375 & 0.4639 & 0.7813 & 1.3114 \\
0.1094 & 0.2166 & 0.4531 & 0.3663 & 0.7969 & 1.5335 \\
0.1250 & 0.2593 & 0.4688 & 0.5287 & 0.8125 & 1.3820 \\
0.1406 & 0.2412 & 0.4844 & 0.5164 & 0.8281 & 1.5679 \\
0.1563 & 0.5773 & 0.5000 & 0.4502 & 0.8438 & 1.4858 \\
0.1719 & 0.4322 & 0.5156 & 0.4488 & 0.8594 & 1.6145 \\
0.1875 & 0.4384 & 0.5313 & 0.4538 & 0.8750 & 1.6282 \\
0.2031 & 0.2872 & 0.5469 & 0.2729 & 0.8906 & 1.7043 \\
0.2188 & 0.3078 & 0.5625 & 0.5069 & 0.9063 & 1.9432 \\
0.2344 & 0.3761 & 0.5781 & 0.6164 & 0.9219 & 1.8384 \\
0.2500 & 0.1896 & 0.5938 & 0.9359 & 0.9375 & 2.1171 \\
0.2656 & 0.1558 & 0.6094 & 0.8222 & 0.9531 & 2.3878 \\
0.2813 & 0.3807 & 0.6250 & 0.7422 & 0.9688 & 2.5204 \\
0.2969 & 0.3637 & 0.6406 & 0.9326 & 0.9844 & 2.7823 \\
0.3125 & 0.3641 & 0.6563 & 1.0095 & 1.0000 & 3.1237 \\
0.3281 & 0.3580 & 0.6719 & 1.0371 & - & - \\
\hline & & & & &
\end{tabular}

TABLE 6 | The data of $X_{t}^{H}$ with $\theta=-100$ and $H=0.5$.

\begin{tabular}{lccccc}
$\boldsymbol{t}$ & $\mathbf{X}_{\mathbf{t}}^{\mathbf{H}}$ & $\boldsymbol{t}$ & $\mathbf{X}_{\mathbf{t}}^{\mathbf{H}}$ & $\boldsymbol{t}$ & $\mathbf{X}_{\mathbf{t}}^{\mathbf{H}}$ \\
\hline 0.0000 & 0.0000 & 0.3438 & 2.1870 & 0.6875 & $5.26 \mathrm{E}+07$ \\
0.0156 & -0.1749 & 0.3594 & 3.5867 & 0.7031 & $1.52 \mathrm{E}+08$ \\
0.0313 & -0.3397 & 0.3750 & 6.3084 & 0.7188 & $4.52 \mathrm{E}+08$ \\
0.0469 & -0.4106 & 0.3906 & 11.0159 & 0.7344 & $1.37 \mathrm{E}+09$ \\
0.0625 & -0.3348 & 0.4063 & 19.5047 & 0.7500 & $4.29 \mathrm{E}+09$ \\
0.0781 & -0.3567 & 0.4219 & 35.6469 & 0.7656 & $1.37 \mathrm{E}+10$ \\
0.0938 & -0.3936 & 0.4375 & 66.9024 & 0.7813 & $4.50 \mathrm{E}+10$ \\
0.1094 & -0.3411 & 0.4531 & 129.1499 & 0.7969 & $1.51 \mathrm{E}+11$ \\
0.1250 & -0.2522 & 0.4688 & 255.5964 & 0.8125 & $5.21 \mathrm{E}+11$ \\
0.1406 & -0.1583 & 0.4844 & 518.9528 & 0.8281 & $1.84 \mathrm{E}+12$ \\
0.1563 & -0.1543 & 0.5000 & $1.08 \mathrm{E}+03$ & 0.8438 & $6.66 \mathrm{E}+12$ \\
0.1719 & 0.0877 & 0.5156 & $2.31 \mathrm{E}+03$ & 0.8594 & $2.47 \mathrm{E}+13$ \\
0.1875 & -0.1242 & 0.5313 & $5.07 \mathrm{E}+03$ & 0.8750 & $9.42 \mathrm{E}+13$ \\
0.2031 & -0.0522 & 0.5469 & $1.14 \mathrm{E}+04$ & 0.8906 & $3.67 \mathrm{E}+14$ \\
0.2188 & 0.1336 & 0.5625 & $2.63 \mathrm{E}+04$ & 0.9063 & $1.47 \mathrm{E}+15$ \\
0.2344 & 0.0243 & 0.5781 & $6.23 \mathrm{E}+04$ & 0.9219 & $6.02 \mathrm{E}+15$ \\
0.2500 & 0.1665 & 0.5938 & $1.51 \mathrm{E}+05$ & 0.9375 & $2.53 \mathrm{E}+16$ \\
0.2656 & 0.2096 & 0.6094 & $3.77 \mathrm{E}+05$ & 0.9531 & $1.09 \mathrm{E}+17$ \\
0.2813 & 0.4085 & 0.6250 & $9.62 \mathrm{E}+05$ & 0.9688 & $4.81 \mathrm{E}+17$ \\
0.2969 & 0.5852 & 0.6406 & $2.52 \mathrm{E}+06$ & 0.9844 & $2.18 \mathrm{E}+18$ \\
0.3125 & 0.8397 & 0.6563 & $6.76 \mathrm{E}+06$ & 1.0000 & $1.01 \mathrm{E}+19$ \\
0.3281 & 1.3366 & 0.6719 & $1.86 \mathrm{E}+07$ & - & -
\end{tabular}

for any $F \in \mathbb{D}^{1,2}$. We have $\mathbb{D}^{1,2} \subset \operatorname{Dom}(\delta)$ and for any $u \in \mathbb{D}^{1,2}$

$$
\begin{aligned}
E\left[\delta(u)^{2}\right] & =E\|u\|_{\mathcal{H}}^{2}+E\langle D u,(D u) *\rangle_{\mathcal{H} \otimes \mathcal{H}} \\
& =E\|u\|_{\mathcal{H}}^{2}+E \int_{[0, T]^{4}} D_{\xi} u_{r} D_{\eta} u_{s} \phi_{H}(\eta, r) \phi_{H}(\xi, s) d s d r d \xi d \eta,
\end{aligned}
$$

where $\left(D^{S} u\right)^{*}$ is the adjoint of $D u$ in the Hilbert space $\mathcal{H} \otimes \mathcal{H}$. We will denote

$$
\delta(u)=\int_{0}^{T} u_{s} \delta S_{s}^{H}
$$

for an adapted process $u$, and it is called Skorohod integral. Alós et al [16], we can obtain the relationship between the Skorohod and Young integral as follows

$$
\int_{0}^{T} u_{s} d S_{s}^{H}=\int_{0}^{T} u_{s} \delta S_{s}^{H}+\int_{0}^{T} \int_{0}^{T} D_{s} u_{t} \psi(t, s) d s d t
$$

provided $u$ has a bounded $q$-variation with $1 \leq q<\frac{1}{H}$ and $u \in \mathbb{D}^{1,2}(\mathcal{H})$ such that

$$
\int_{0}^{T} \int_{0}^{T} D_{s} u_{t} \psi(t, s) d s d t<\infty
$$

Theorem 2.1. (Alós et al [16]). Let $0<H<1$ and let $f \in C^{2}(\mathbb{R})$ such that

$$
\max \left\{|f(x)|,\left|f^{\prime}(x)\right|,\left|f^{\prime \prime}(x)\right|\right\} \leq \kappa e^{\beta x^{2}},
$$

where $\kappa$ and $\beta$ are two positive constants with $\beta<\frac{1}{4} T^{-2 H}$. Then we have

$$
\begin{aligned}
f\left(S_{t}^{H}\right)=f(0)+ & \int_{0}^{t} f^{\prime}\left(S_{s}^{H}\right) d S_{s}^{H}+H\left(2-2^{2 H-1}\right) \\
& \int_{0}^{t} f^{\prime \prime}\left(S_{s}^{H}\right) s^{2 H-1} d s
\end{aligned}
$$

for all $t \in[0, T]$.

\section{SOME BASIC ESTIMATES}

Throughout this paper we assume that $\theta<0$ and $\frac{1}{2}<H<1$. Recall that the linear self-interacting diffusion with sub-fBm $S^{H}$ defined by the stochastic differential equation

$$
X_{t}^{H}=S_{t}^{H}-\theta \int_{0}^{t} \int_{0}^{s}\left(X_{s}^{H}-X_{u}^{H}\right) d u d s+v t, \quad t \geq 0
$$

with $\theta<0$. Define the kernel $(t, s) \mapsto h_{\theta}(t, s)$ as follows

$$
h_{\theta}(t, s)= \begin{cases}1-\theta s e^{\frac{1}{2} \theta s^{2}} \int_{s}^{t} e^{-\frac{1}{2} \theta u^{2}} d u, & t \geq s, \\ 0, & t<s\end{cases}
$$

for $s, t \geq 0$. By the variation of constants method (see, Cranston and Le Jan [1]) or Itô's formula we may introduce the following representation:

$$
X_{t}^{H}=\int_{0}^{t} h_{\theta}(t, s) d S_{s}^{H}+v \int_{0}^{t} h_{\theta}(t, s) d s
$$

for $t \geq 0$.

The kernel function $(t, s) \mapsto h_{\theta}(t, s)$ with $\theta<0$ admits the following properties (these properties are proved partly in Sun and Yan [12]):

- For all $s \geq 0$, the limit

$$
\lim _{t \rightarrow \infty}\left(t e^{\frac{1}{2} \theta t^{2}} h_{\theta}(t, s)\right)=s e^{\frac{1}{2} \theta s^{2}}
$$

for all $s \geq 0$. 
- For all $t \geq s \geq 0$, we have

$$
1 \leq h_{\theta}(t, s) \leq e^{-\frac{1}{2} \theta\left(t^{2}-s^{2}\right)}
$$

- For all $t \geq s, r \geq 0$, we have

$$
h_{\theta}(t, 0)=h_{\theta}(t, t)=1, \quad \int_{s}^{t} h_{\theta}(t, u) d u=e^{\frac{1}{2} \theta s^{2}} \int_{s}^{t} e^{-\frac{1}{2} \theta u^{2}} d u .
$$

Lemma 3.1. Let $\theta<0$ and define function

$$
I_{\theta}(t)=-\theta t e^{\frac{1}{2} \theta t^{2}} \int_{0}^{t} e^{-\frac{1}{2} \theta u^{2}} d u-1 .
$$

We then have $\lim _{t \rightarrow \infty} t^{2} I_{\theta}(t)=-\frac{1}{\theta}$ and

$$
\lim _{t \rightarrow \infty} t^{2}\left(1+\theta t e^{-\frac{1}{2} \theta t^{2}} \int_{t}^{\infty} e^{\frac{1}{2} \theta u^{2}} d u\right)=-\frac{1}{\theta}
$$

Proof. This is simple calculus exercise.

Lemma 3.2. (Sun and Yan [12]). Let $\theta<0$ and define the functions $t \mapsto I_{\theta}(t, n), n=1,2, \ldots$ as follows

$I_{\theta}(t, 1)=-\theta t^{2} I_{\theta}(t), \quad I_{\theta}(t, n+1)=-\theta t^{2}\left[I_{\theta}(t, n)-(2 n-1) ! !\right]$.

Then we have

$$
\lim _{t \rightarrow \infty} I_{\theta}(t, n)=(2 n-1) ! ! .
$$

for every $n \geq 0$, where $(-1) !=1$.

Lemma 3.3. Let $\theta<0$. Then the integral

$$
\Delta(H)=\int_{0}^{\infty} \int_{0}^{\infty} x y e^{\frac{1}{2} \theta\left(x^{2}+y^{2}\right)} \psi_{H}(x, y) d x d y
$$

converges and as $t \rightarrow \infty$,

$$
\lim _{t \rightarrow \infty} t^{2} e^{-\theta t^{2}} E\left(X_{t}^{H}\right)^{2}=\Delta(H) .
$$

Proof. An elementary may show that (3.6) converges for all $\theta<0$. It follows from L'Hôspital's rule that

$$
\begin{aligned}
\lim _{t \rightarrow \infty} & t^{2} e^{\theta t^{2}} E\left(X_{t}^{H}\right)^{2}=\lim _{t \rightarrow \infty} t^{2} e^{\theta t^{2}} \int_{0}^{t} \int_{0}^{t} h_{\theta}(t, x) h_{\theta}(t, y) \psi_{H}(x, y) d x d y \\
& =\lim _{t \rightarrow \infty} \frac{\theta^{2}}{t^{-2} e^{-\theta t^{2}}} \int_{0}^{t} d x \int_{0}^{t} x y e^{\frac{1}{2} \theta\left(x^{2}+y^{2}\right)} \psi_{H}(x, y) d y \int_{x}^{t} d u \int_{y}^{t} e^{-\frac{1}{2} \theta\left(u^{2}+v^{2}\right)} d v \\
& =2 \lim _{t \rightarrow \infty} \frac{\theta^{2}}{t^{-2} e^{-\theta t^{2}}} \int_{0}^{t} d u \int_{0}^{u} d x \int_{0}^{u} d v \int_{0}^{v} d y x y e^{\frac{1}{2} \theta\left(x^{2}+y^{2}-u^{2}-v^{2}\right)} \psi_{H}(x, y) \\
& =\lim _{t \rightarrow \infty} \frac{-\theta}{t^{-1} e^{-\frac{1}{2} \theta t^{2}}} \int_{0}^{t} d x \int_{0}^{t} e^{-\frac{1}{2} \theta v^{2}} d v \int_{0}^{v} x y e^{\frac{1}{2} \theta\left(x^{2}+y^{2}\right)} \psi_{H}(x, y) d y \\
& =\lim _{t \rightarrow \infty} \frac{-\theta}{t^{-1} e^{-\frac{1}{2} \theta t^{2}}} \int_{0}^{t} e^{-\frac{1}{2} \theta v^{2}} d v \int_{0}^{t} d x \int_{0}^{v} x y e^{\frac{1}{2} \theta\left(x^{2}+y^{2}\right)} \psi_{H}(x, y) d y \\
& =\lim _{t \rightarrow \infty} \frac{-\theta}{t^{-1} e^{-\frac{1}{2} \theta t^{2}}} \int_{0}^{t} e^{-\frac{1}{2} \theta v^{2}} d v \int_{0}^{v} d x \int_{0}^{v} x y e^{\frac{1}{2} \theta\left(x^{2}+y^{2}\right)} \psi_{H}(x, y) d y \\
& =\int_{0}^{\infty} d x \int_{0}^{\infty} x y e^{\frac{1}{2} \theta\left(x^{2}+y^{2}\right)} \psi_{H}(x, y) d y,
\end{aligned}
$$

where we have used the following fact:

$$
\begin{gathered}
\lim _{t \rightarrow \infty} \frac{1}{t^{-1} e^{-\frac{1}{2} \theta t^{2}}} \int_{0}^{t} e^{-\frac{1}{2} \theta v^{2}} d v \int_{v}^{t} d x \int_{0}^{v} x y e^{\frac{1}{2} \theta\left(x^{2}+y^{2}\right)} \psi_{H}(x, y) d y \\
=\lim _{t \rightarrow \infty} \frac{1}{t^{-1} e^{-\frac{1}{2} \theta t^{2}}} \int_{0}^{t} d x \int_{0}^{x} e^{-\frac{1}{2} \theta v^{2}} d v \\
\int_{0}^{v} x y e^{\frac{1}{2} \theta\left(x^{2}+y^{2}\right)} \psi_{H}(x, y) d y=0 .
\end{gathered}
$$

This completes the proof.

Lemma 3.4. Let $\theta<0$. Then, convergence

$$
\begin{aligned}
& \lim _{t \rightarrow \infty} \frac{1}{t^{2-2 H}} e^{-\theta t^{2}} \int_{t}^{\infty} \int_{s}^{\infty} s r e^{\frac{1}{2} \theta\left(s^{2}+r^{2}\right)} \psi_{H}(s, r) d s d r \\
& \quad=\frac{1}{4}(-\theta)^{-2 H} \Gamma(2 H+1) .
\end{aligned}
$$

holds.

Proof. It follows from L'Hôspital's rule that

$$
\begin{aligned}
\lim _{t \rightarrow \infty} & \frac{1}{t^{2-2 H} e^{\theta t^{2}}} \int_{t}^{\infty} u e^{\frac{1}{2} \theta u^{2}}\left(\int_{u}^{\infty} v e^{\frac{1}{2} \theta v^{2}} \psi_{H}(u, v) d v\right) d u \\
& =-\frac{1}{2 \theta} \lim _{t \rightarrow \infty} \frac{1}{t^{2-2 H} e^{\frac{1}{2} \theta t^{2}}} \int_{t}^{\infty} v e^{\frac{1}{2} \theta v^{2}} \psi_{H}(t, v) d v \\
& =-\lim _{t \rightarrow \infty} \frac{H(2 H-1)}{2 \theta t^{2-2 H}} \int_{t}^{\infty} v e^{\frac{1}{2} \theta\left(v^{2}-t^{2}\right)}\left((v-t)^{2 H-2}-(v+t)^{2 H-2}\right) d v
\end{aligned}
$$

for all $\theta<0$ and $\frac{1}{2}<H<1$. By making the change of variable $\frac{1}{2} \theta\left(v^{2}-t^{2}\right)=x$, we see that

$$
\begin{gathered}
\lim _{t \rightarrow \infty} \frac{1}{2 \theta t^{2-2 H}} \int_{t}^{\infty} v e^{\frac{1}{2} \theta\left(v^{2}-t^{2}\right)}\left((v-t)^{2 H-2}-(v+t)^{2 H-2}\right) d v \\
=\lim _{t \rightarrow \infty} \frac{1}{2 \theta^{2} t^{2-2 H}} \int_{0}^{\infty} e^{-x}\left\{\left(\sqrt{\left.t^{2}+\frac{2 x}{-\theta}-t\right)^{2 H-2}}\right.\right. \\
\left.\quad-\left(\sqrt{t^{2}+x}+t\right)^{2 H-2}\right\} d x \\
=\lim _{t \rightarrow \infty} \frac{1}{2 \theta^{2} t^{2-2 H}} \int_{0}^{\infty} e^{-x}\left(\frac{2 x}{-\theta}\right)^{2 H-2}\left(\sqrt{t^{2}+\frac{2 x}{-\theta}}+t\right)^{2-2 H} d x \\
\quad-\lim _{t \rightarrow \infty} \frac{1}{2 \theta^{2} t^{2-2 H}} \int_{0}^{\infty} e^{-x}\left(\sqrt{t^{2}+x}+t\right)^{2 H-2} d x \\
=\frac{1}{2}(-\theta)^{-2 H-1} \Gamma(2 H-1)
\end{gathered}
$$

for all $\theta<0$ and $\frac{1}{2}<H<1$. This completes the proof.

Lemma 3.5. Let $\theta<0$ and $0 \leq s<t \leq T$. We then have

$$
c(t-s)^{2 H} \leq E\left[\left(X_{t}^{H}-X_{s}^{H}\right)^{2}\right] \leq C(t-s)^{2 H}
$$

Proof. Given $0 \leq s<t \leq T$ and denote

$$
\hat{X}_{t}^{H}=\int_{0}^{t} h_{\theta}(t, r) d S_{r}^{H}, \quad t \geq 0 .
$$


It follows that

$$
\begin{aligned}
E\left[\left(\hat{X}_{t}^{H}-\hat{X}_{s}^{H}\right)^{2}\right]= & E\left(\int_{0}^{s}\left[h_{\theta}(t, x)-h_{\theta}(s, x)\right] d S_{x}^{H}\right)^{2} \\
& +E\left(\int_{s}^{t} h_{\theta}(t, x) d S_{x}^{H}\right)^{2} \\
& +2 E\left(\int_{s}^{t} h_{\theta}(t, y) d S_{y}^{H} \int_{0}^{s}\left[h_{\theta}(t, x)-h_{\theta}(s, x)\right] d S_{x}^{H}\right) .
\end{aligned}
$$

Now, we estimate the three terms. For the first term, we have

$$
\begin{aligned}
0 \leq & E\left(\int_{0}^{s}\left[h_{\theta}(t, x)-h_{\theta}(s, x)\right] d S_{x}^{H}\right)^{2} \\
& =\int_{0}^{s} \int_{0}^{s}\left(h_{\theta}(t, x)-h_{\theta}(s, x)\right) \\
& \left(h_{\theta}(t, y)-h_{\theta}(s, y)\right) \psi_{H}(x, y) d x d y \\
& =\theta^{2}\left(\int_{s}^{t} e^{-\frac{1}{2} \theta u^{2}} d u\right)^{2} \int_{0}^{s} \int_{0}^{s} x y e^{\frac{1}{2} \theta\left(x^{2}+y^{2}\right)} \psi_{H}(x, y) d x d y \\
& \leq \theta^{2} s^{2}(t-s)^{2} e^{-\theta t^{2}} \int_{0}^{s} \int_{0}^{s} \psi_{H}(x, y) d x d y \\
& =\theta^{2} s^{2}(t-s)^{2} e^{-\theta t^{2}} E\left(S_{s}^{H}\right)^{2} \leq C_{H, T}(t-s)^{2}
\end{aligned}
$$

for all $\theta<0$ and $0<s<t \leq T$. For the second term, we have

$$
\begin{aligned}
E\left(\int_{s}^{t} h_{\theta}(t, x) d S_{x}^{H}\right)^{2} & =\int_{s}^{t} \int_{s}^{t} h_{\theta}(t, x) h_{\theta}(t, y) \psi_{H}(x, y) d x d y \\
& \leq e^{-\theta t^{2}} \int_{s}^{t} \int_{s}^{t} x y e^{\frac{1}{2} \theta\left(x^{2}+y^{2}\right)} \psi_{H}(x, y) d x d y \\
& \leq t^{2} e^{-\theta t^{2}} \int_{s}^{t} \int_{s}^{t} \psi_{H}(x, y) d x d y \\
& \leq C_{H, T}(t-s)^{2 H}
\end{aligned}
$$

for all $\theta<0$ and $0<s<t \leq T$. Similarly, for the third term, we also prove

$$
\begin{aligned}
0 \leq E & \left(\int_{s}^{t} h_{\theta}(t, y) d S_{y}^{H} \int_{0}^{s}\left[h_{\theta}(t, x)-h_{\theta}(s, x)\right] d S_{x}^{H}\right) \\
& =\int_{s}^{t} \int_{0}^{s} h_{\theta}(t, y)\left[h_{\theta}(t, x)-h_{\theta}(s, x)\right] \psi_{H}(x, y) d x d y \\
& \leq \theta^{2} e^{-\frac{1}{2} \theta t^{2}}\left(\int_{s}^{t} e^{-\frac{1}{2} \theta u^{2}} d u\right) \int_{s}^{t} y e^{\frac{1}{2} \theta y^{2}} d y \int_{0}^{s} x e^{\frac{1}{2} \theta x^{2}} \psi_{H}(x, y) d x \\
& \leq \theta^{2} e^{-\theta t^{2}}(t-s) \int_{s}^{t} y e^{\frac{1}{2} \theta y^{2}} d y \int_{0}^{s} x e^{\frac{1}{2} \theta x^{2}} \psi_{H}(x, y) d x \\
& \leq C_{H, T}(t-s)^{2}
\end{aligned}
$$

for all $\theta<0$ and $0<s<t \leq T$. Thus, we have obtained the following estimate:

$$
E\left[\left(\hat{X}_{t}^{H}-\hat{X}_{s}^{H}\right)^{2}\right] \leq C_{H, T}|t-s|^{2 H}
$$

for all $\theta<0$ and $0<s<t \leq T$.

On the other hand, elementary calculations may show that $\int_{0}^{s}\left[h_{\theta}(t, r)-h_{\theta}(s, r)\right] d r=\theta \int_{s}^{t} e^{-\frac{1}{2} \theta u^{2}} d u \int_{0}^{s} r e^{\frac{1}{2} \theta r^{2}} d r \leq C_{H, T}(t-s)$

and

$$
\int_{s}^{t} h_{\theta}(t, r) d r=e^{-\frac{1}{2} \theta s^{2}} \int_{s}^{t} e^{\frac{1}{2} \theta r^{2}} d r \leq C_{H, T}(t-s)
$$

for all $\theta<0$ and $0<s<t \leq T$. It follows that

$$
\begin{gathered}
\left(\int_{0}^{t} h_{\theta}(t, r) d r-\int_{0}^{s} h_{\theta}(s, r) d r\right)^{2} \\
=\left(\int_{0}^{s}\left[h_{\theta}(t, r)-h_{\theta}(s, r)\right] d r\right)^{2} \\
+\left(\int_{s}^{t} h_{\theta}(t, r) d r\right)^{2}+2 \int_{s}^{t} h_{\theta}(t, r) d r \int_{0}^{s}\left[h_{\theta}(t, r)-h_{\theta}(s, r)\right] d r \\
\leq C_{H, T}(t-s)^{2}
\end{gathered}
$$

for all $\theta<0$ and $0<s<t \leq T$, which implies that

$$
\begin{aligned}
E\left[\left(X_{t}^{a, b}-X_{s}^{a, b}\right)^{2}\right]= & E\left[\left(\hat{X}_{t}^{a, b}-\hat{X}_{s}^{a, b}\right)^{2}\right] \\
& +v^{2}\left(\int_{0}^{t} h_{\theta}(t, r) d r-\int_{0}^{s} h_{\theta}(s, r) d r\right)^{2} \\
\leq & C_{H, T}(t-s)^{2 H}
\end{aligned}
$$

for all $\theta<0$ and $0<s<t \leq T$. Noting that the above calculations are invertible for all $\theta<0$ and $0<s<t \leq T$, one can obtain the left hand side in (3.8) and the lemma follows.

\section{CONVERGENCE}

In this section, we obtain the large time behaviors associated with the solution $X^{H}$ to Eq. 3.1. From Lemma 3.5 and Guassianness, we find that the self-repelling diffusion $\left\{X_{t}^{H}, t \geq 0\right\}$ is $H$-Hölder continuous. So, the integral

$$
\int_{0}^{t} s d X_{s}^{H}
$$

exists with $t \geq 0$ as a Young integral and

$$
t X_{t}^{H}=\int_{0}^{t} s d X_{s}^{H}+\int_{0}^{t} X_{s}^{H} d s
$$

for all $t \geq 0$. Define the process $Y=\left\{Y_{t}, t \geq 0\right\}$ by

$$
\begin{aligned}
Y_{t}: & =\int_{0}^{t}\left(X_{t}^{H}-X_{s}^{H}\right) d s=t X_{t}^{H}-\int_{0}^{t} X_{s}^{H} d s=\int_{0}^{t} s d X_{s}^{H} \\
& =\int_{0}^{t} s d S_{s}^{H}-\int_{0}^{t} \theta s Y_{s} d s+\frac{1}{2} v t^{2} .
\end{aligned}
$$

By the variation of constants method, one can prove

$$
Y_{t}=e^{-\frac{1}{2} \theta t^{2}} \int_{0}^{t} s e^{\frac{1}{2} \theta s^{2}} d S_{s}^{H}-\frac{\nu}{\theta}\left(e^{-\frac{1}{2} \theta t^{2}}-1\right)
$$

for all $t \geq 0$. Define Gaussian process $\xi^{H}=\left\{\xi_{t}^{H}, t \geq 0\right\}$ as follows

$$
\xi_{t}^{H}:=\int_{0}^{t} s e^{\frac{1}{2} \theta s^{2}} d S_{s}^{H}, \quad t \geq 0 .
$$


Lemma 4.1. Let $\theta<0$ and $\frac{1}{2}<H<1$. Then, the random variable

$$
\xi_{\infty}^{H}:=\int_{0}^{\infty} s e^{\frac{1}{2} \theta s^{2}} d S_{s}^{H}
$$

exists as an element in $L^{2}$. Moreover, $\xi^{H}$ is H-Hölder continuous and $\xi_{t}^{H} \rightarrow \xi_{\infty}^{H}$ in $L^{2}$ and almost surely, as $t$ tends to infinity.

Proof. This is simple calculus exercise. In fact, we have

$$
\begin{aligned}
E\left(\int_{0}^{\infty} x e^{\frac{1}{2} \theta x^{2}} d S_{x}^{H}\right)^{2} & =\int_{0}^{\infty} \int_{0}^{\infty} x y e^{\frac{1}{2} \theta\left(x^{2}+y^{2}\right)} \psi_{H}(x, y) d x d y \\
& =2 \int_{0}^{\infty} x e^{\frac{1}{2} \theta x^{2}} d x \int_{0}^{x} y e^{\frac{1}{2} \theta y^{2}} \psi_{H}(x, y) d y \\
& =2 H(2 H-1) \int_{0}^{\infty} x e^{\frac{1}{2} \theta x^{2}} d x \\
& \int_{0}^{x} y e^{\frac{1}{2} \theta y^{2}}\left((x-y)^{2 H-2}-(x+y)^{2 H-2}\right) d y \\
& \leq 2 H(2 H-1) \int_{0}^{\infty} x e^{\frac{1}{2} \theta x^{2}} d x \\
& \int_{0}^{x}\left((x-y)^{2 H-2}-(x+y)^{2 H-2}\right) y d y \\
& =2 H(2 H-1) C_{H} \int_{0}^{\infty} x^{2 H+1} e^{\frac{1}{2} \theta x^{2}} d x \\
& =C_{\theta, H} \Gamma(2 H+2)
\end{aligned}
$$

for all $\theta<0$ and $\frac{1}{2}<H<1$, which shows that the random variable $\xi_{\infty}^{H}$ exists as an element in $L^{2}$.

Now, we show that the process $\xi^{a, b}$ is Hölder continuous. For all $0<s<t$ by the inequality $e^{-x^{2}} x \leq C$ for all $x \geq 0$, we have

$$
\begin{aligned}
E\left(\xi_{t}^{H}-\xi_{s}^{H}\right)^{2} & =E\left(\int_{s}^{t} x e^{\frac{1}{2} \theta x^{2}} d S_{x}^{H}\right)^{2} \\
& =\int_{s}^{t} \int_{s}^{t} x y e^{\frac{1}{2} \theta\left(x^{2}+y^{2}\right)} \psi_{H}(x, y) d x d y \\
& =2 \int_{s}^{t} x e^{\frac{1}{2} \theta x^{2}} d x \int_{s}^{x} y e^{\frac{1}{2} \theta y^{2}} \psi_{H}(x, y) d y \\
& =2 H(2 H-1) \int_{s}^{t} x e^{\frac{1}{2} \theta x^{2}} d x \int_{s}^{x} y e^{\frac{1}{2} \theta y^{2}}\left((x-y)^{2 H-2}\right. \\
& \left.-(x+y)^{2 H-2}\right) d y \\
\leq & 2 H C_{\theta}(2 H-1) \int_{s}^{t} d x \int_{s}^{x}(x-y)^{2 H-2} d y \\
& =C_{\theta, H}(t-s)^{2 H} .
\end{aligned}
$$

Thus, the normality of $\xi^{H}$ implies that

$$
E\left(\xi_{t}^{H}-\xi_{s}^{H}\right)^{2 n} \leq C_{\theta, H, n}(t-s)^{2 n H}
$$

for all $0 \leq s<t, \frac{1}{2}<H<1$ and integer numbers $n \geq 1$, and the Hölder continuity follows.
Nextly, we check the $\xi_{t}^{a, b}$ converges to $\xi_{\infty}^{H}$ in $L^{2}$. This follows from the next estimate:

$$
\begin{aligned}
& E\left(\xi_{t}^{H}-\xi_{\infty}^{H}\right)^{2}=\int_{t}^{\infty} \int_{t}^{\infty} x y e^{\frac{1}{2} \theta\left(x^{2}+y^{2}\right)} \psi_{H}(x, y) d x d y \\
&=2 \int_{t}^{\infty} \int_{t}^{x} x y e^{\frac{1}{2} \theta\left(x^{2}+y^{2}\right)} \psi_{H}(x, y) d x d y \\
& \leq 2 e^{\frac{1}{2} \theta t^{2}} \int_{t}^{\infty} x e^{\frac{1}{2} \theta x^{2}} d x \int_{t}^{x} y \psi_{H}(x, y) d y \\
& \leq 2 e^{\frac{1}{2} \theta t^{2}} \int_{t}^{\infty} x e^{\frac{1}{2} \theta x^{2}} d x \int_{0}^{x} y \psi_{H}(x, y) d y \\
& \leq 2 H(2 H-1) e^{\frac{1}{2} \theta t^{2}} \\
& \cdot \int_{t}^{\infty} x e^{\frac{1}{2} \theta x^{2}} d x \int_{0}^{x} y\left((x-y)^{2 H-2}\right. \\
&\left.-(x+y)^{2 H-2}\right) d y \\
& \leq 2 H(2 H-1) e^{\frac{1}{2} \theta t^{2}} \cdot \int_{t}^{\infty} x e^{\frac{1}{2} \theta x^{2}} d x \int_{0}^{x} y(x-y)^{2 H-2} d y \\
&=2 H(2 H-1)\left(\int_{0}^{1} u(1-u)^{2 H-2} d u\right) e^{\frac{1}{2} \theta t^{2}} \\
& \int_{t}^{\infty} x^{2 H+1} e^{\frac{1}{2} \theta x^{2}} d x \rightarrow 0,
\end{aligned}
$$

as $t$ tends to infinity.

Finally, we check the $\xi_{t}^{a, b}$ converges to $\xi_{\infty}^{H}$ almost surely. By integration by parts we see that

$$
\xi_{t}^{H}-\xi_{\infty}^{H}=\int_{t}^{\infty} s e^{\frac{1}{2} \theta s^{2}} d S_{s}^{H}=-t e^{\frac{1}{2} \theta t^{2}} S_{t}^{H}-\int_{t}^{\infty}\left(1+\theta s^{2}\right) e^{\frac{1}{2} \theta s^{2}} S_{s}^{H} d s
$$

for all $t \geq 0$. Elementary may check that the convergence

$$
\eta_{t}^{H}:=\int_{t}^{\infty}\left(1+\theta s^{2}\right) e^{\frac{1}{2} \theta s^{2}} S_{s}^{H} d s \stackrel{a . s}{\rightarrow} 0
$$

holds almost surely, as $t$ tends to infinity. In fact, by inequality

$$
\int_{t}^{\infty} s^{\alpha} e^{\frac{1}{2} \theta s^{2}} d s \leq C t^{\alpha-1} e^{\frac{1}{2} \theta t^{2}}, \quad \alpha>-1,
$$

with $t \geq 0$, we may show that

$$
\begin{gathered}
E\left(\sup _{n \leq t<n+1}\left|\eta_{t}^{H}\right|^{2}\right) \leq \int_{n}^{\infty} \int_{n}^{\infty}\left(1+\theta s^{2}\right) \\
\left(1+\theta r^{2}\right) e^{\frac{1}{2} \theta\left(s^{2}+r^{2}\right)} E\left|S_{s}^{H} \| S_{r}^{H}\right| d r d s \\
\leq C\left(\int_{n}^{\infty} s^{2+H} e^{\frac{1}{2} \theta s^{2}} d s\right)^{2} \\
\leq C n^{2+2 H} e^{\theta n^{2}},
\end{gathered}
$$

for all integer numbers $n \geq 1$, and hence 


$$
\sum_{n=0}^{\infty} P\left(\sup _{n \leq t<n+1}\left|\eta_{t}^{H}\right|^{2} \geq \varepsilon\right) \leq C \varepsilon^{-2} \sum_{n=0}^{\infty} n^{2+2 H} e^{\theta n^{2}}<\infty .
$$

Thus, Borel-Cantelli's lemma implies that $\eta_{t}^{H}$ converges to zero almost surely as $t$ tends to infinity, and the lemma follows from (4.2).

Corollary 4.1. For all $\gamma>0$, we have

$$
t^{\gamma}\left(\xi_{t}^{H}-\xi_{\infty}^{H}\right)=t^{\gamma} \int_{t}^{\infty} s e^{\frac{1}{2} \theta s^{2}} d S_{s}^{H} \rightarrow 0
$$

in $L^{2}$ and almost surely, as $t$ tends to infinity.

Lemma 4.2. Let $\theta<0$ and $\frac{1}{2}<H<1$. Then, we have

$$
\Lambda_{\gamma}(t, \theta):=t^{\gamma+1} e^{\frac{1}{2} \theta t^{2}} \int_{0}^{t} e^{-\frac{1}{2} \theta u^{2}}\left(\xi_{\infty}^{a, b}-\xi_{u}^{a, b}\right) d u \rightarrow 0
$$

in $L^{2}$ and almost surely for every $\gamma \geq 0$, as $t$ tends to infinity.

Proof. Given $0<s \leq t, \theta<0$ and denote

$$
\begin{aligned}
\Upsilon_{\theta}(s, t):= & \int_{0}^{t} e^{-\frac{1}{2} \theta v^{2}} d v \int_{v}^{\infty} r e^{\frac{1}{2} \theta r^{2}} \psi_{H}(s, r) d r \\
= & \int_{0}^{t} r e^{\frac{1}{2} \theta r^{2}} \psi_{H}(s, r) d r \int_{0}^{r} e^{-\frac{1}{2} \theta v^{2}} d v \\
& +\left(\int_{0}^{t} e^{-\frac{1}{2} \theta v^{2}} d v\right) \int_{t}^{\infty} r e^{\frac{1}{2} \theta r^{2}} \psi_{H}(s, r) d r \\
\leq & C \int_{0}^{t} r \psi_{H}(s, r) d r+\frac{C}{t} e^{-\frac{1}{2} \theta t^{2}} \int_{t}^{\infty} r e^{\frac{1}{2} \theta r^{2}} \psi_{H}(s, r) d r \\
\leq & C\left(\int_{0}^{t} r \psi_{H}(s, r) d r+(t-s)^{2 H-2} t^{-1}\right),
\end{aligned}
$$

where we have used the fact

$$
\int_{0}^{x} e^{-\frac{1}{2} \theta v^{2}} d v \leq \frac{C}{x} e^{-\frac{1}{2} \theta x^{2}}, \quad \forall x \geq 0
$$

and estimates

$$
\begin{aligned}
\int_{t}^{\infty} r e^{\frac{1}{2} \theta r^{2}} \psi_{H}(s, r) d r= & H(2 H-1) \int_{t}^{\infty} r\left((r-s)^{2 H-2}\right. \\
& \left.-(s+r)^{2 H-2}\right) e^{\frac{1}{2} \theta r^{2}} d r \\
\leq & H(2 H-1) \int_{t}^{\infty} r(r-s)^{2 H-2} e^{\frac{1}{2} \theta r^{2}} d r \\
\leq & H(2 H-1)(t-s)^{2 H-2} \int_{t}^{\infty} r e^{\frac{1}{2} \theta r^{2}} d r \\
= & \frac{H(2 H-1)}{-\theta}(t-s)^{2 H-2} e^{\frac{1}{2} \theta t^{2}} .
\end{aligned}
$$

It follows that

$$
\begin{aligned}
E\left|\Lambda_{\gamma}(t, \theta)\right|^{2}= & t^{2 \gamma+2} e^{\theta t^{2}} \int_{0}^{t} \int_{0}^{t} e^{-\frac{1}{2} \theta\left(u^{2}+v^{2}\right)} \\
& \cdot E\left(\int_{u}^{\infty} s e^{\frac{1}{2} \theta s^{2}} d S_{s}^{H}\right)\left(\int_{v}^{\infty} r e^{\frac{1}{2} \theta r^{2}} d S_{r}^{H}\right) d u d v \\
= & t^{2 \gamma+2} e^{\theta t^{2}} \int_{0}^{t} \int_{0}^{t} e^{-\frac{1}{2} \theta\left(u^{2}+v^{2}\right)} d u d v \\
& \int_{u}^{\infty} \int_{v}^{\infty} r s e^{\frac{1}{2} \theta\left(r^{2}+s^{2}\right)} \psi_{H}(s, r) d r d s \\
= & t^{2 \gamma+2} e^{\theta t^{2}} \int_{0}^{t} e^{-\frac{1}{2} \theta u^{2}} d u \int_{u}^{\infty} s e^{\frac{1}{2} \theta s^{2}} \Upsilon_{\theta}(s, t) d s \\
= & t^{2 \gamma+2} e^{\theta t^{2}} \int_{0}^{t} s e^{\frac{1}{2} \theta s^{2}} \psi_{H}(s, \theta) d s \int_{0}^{s} e^{-\frac{1}{2} \theta u^{2}} d u \\
& +t^{2 \gamma+2} e^{\theta t^{2}} \int_{t}^{\infty} s e^{\frac{1}{2} \theta s^{2}} \Upsilon_{\theta}(s, t) d s \int_{0}^{t} e^{-\frac{1}{2} \theta u^{2}} d u \\
\leq & t^{2 \gamma+2} e^{\theta t^{2}} \int_{0}^{t} s^{2} \Upsilon_{\theta}(s, t) d s \\
& +t^{2 \gamma+1} e^{\frac{1}{2} \theta t^{2}} \int_{t}^{\infty} s e^{\frac{1}{2} \theta s^{2}} \Upsilon_{\theta}(s, t) d s \\
\rightarrow & 0 \quad(t \rightarrow \infty)
\end{aligned}
$$

which shows that $\Lambda_{\gamma}(t, \theta)$ converges to zero in $L^{2}$.

Now, we obtain the convergence with probability one. Noting that

$$
\xi_{\infty}^{H}-\xi_{u}^{H}=\int_{u}^{\infty} s e^{\frac{1}{2} \theta s^{2}} d S_{s}^{H}
$$

for all $u \geq 0$, we get

$$
\begin{aligned}
& \left|\Lambda_{\gamma}(t, \theta)\right| \leq t^{\gamma+1} e^{\frac{1}{2} \theta t^{2}} \int_{0}^{t} e^{-\frac{1}{2} \theta u^{2}}\left|\int_{u}^{\infty} s e^{\frac{1}{2} \theta s^{2}} d S_{s}^{H}\right| d u \\
& \leq t^{\gamma+1} e^{\frac{1}{2} \theta t^{2}} \int_{0}^{t} e^{-\frac{1}{2} \theta u^{2}}\left(u\left|S_{u}^{H}\right| e^{\frac{1}{2} \theta u^{2}}+\int_{u}^{\infty}\left|S_{s}^{H}\left(1-\theta s^{2}\right)\right| e^{\frac{1}{2} \theta s^{2}} d s\right) d u \\
& =t^{\gamma+1} e^{\frac{1}{2} \theta t^{2}} \int_{0}^{t} u\left|S_{u}^{H}\right| d u+t^{\gamma+1} e^{\frac{1}{2} \theta t^{2}} \int_{0}^{t} e^{-\frac{1}{2} \theta u^{2}} d u \\
& \quad \int_{u}^{\infty}\left|S_{s}^{H}\left(1-\theta s^{2}\right)\right| e^{\frac{1}{2} \theta s^{2}} d s \\
& =t^{\gamma+1} e^{\frac{1}{2} \theta t^{2}} \int_{0}^{t} u\left|S_{u}^{H}\right| d u \\
& \quad+t^{\gamma+1} e^{\frac{1}{2} \theta t^{2}} \int_{0}^{t}\left|S_{s}^{H}\left(1-\theta s^{2}\right)\right| e^{\frac{1}{2} \theta s^{2}} d s \int_{0}^{s} e^{-\frac{1}{2} \theta u^{2}} d u \\
& +t^{\gamma+1} e^{\frac{1}{2} \theta t^{2}} \int_{t}^{\infty}\left|S_{s}^{H}\left(1-\theta s^{2}\right)\right| e^{\frac{1}{2} \theta s^{2}} d s \int_{0}^{t} e^{-\frac{1}{2} \theta u^{2}} d u \\
& \leq t^{\gamma+1} e^{\frac{1}{2} \theta t^{2}} \int_{0}^{t} u\left|S_{u}^{H}\right| d u+t^{\gamma+1} e^{\frac{1}{2} \theta t^{2}} \int_{0}^{t}\left|S_{s}^{H}\left(1-\theta s^{2}\right)\right| s d s \\
& +C_{\theta} t^{\gamma} \int_{t}^{\infty}\left|S_{s}^{H}\left(1-\theta s^{2}\right)\right| e^{\frac{1}{2} \theta s^{2}} d s \\
& \rightarrow 0
\end{aligned}
$$

almost surely for all $\gamma \geq 0, \theta<0$ and $\frac{1}{2}<H<1$, as $t$ tends to infinity. This completes the proof. 
The objects of this paper are to prove the following theorems which give the long time behaviors for $X^{H}$ with $\frac{1}{2}<H<1$.

Theorem 4.1. Let $\theta<0$ and $\frac{1}{2}<H<1$. Then, as $t \rightarrow \infty$, the convergence

$$
J_{0}^{H}(t ; \theta, v):=t e^{\frac{1}{2} \theta t^{2}} X_{t}^{H} \rightarrow \xi_{\infty}^{H}-\frac{v}{\theta}
$$

holds in $L^{2}$ and almost surely.

Proof. Given $t>0$ and $\theta<0$. Simple calculations may prove

$$
\begin{aligned}
J_{0}^{H}(t ; \theta, v)= & t e^{\frac{1}{2} \theta t^{2}} X_{t}^{H} \\
= & t e^{\frac{1}{2} \theta t^{2}} \int_{0}^{t} h_{\theta}(t, s) d S_{s}^{H}+v t e^{\frac{1}{2} \theta t^{2}} \int_{0}^{t} h_{\theta}(t, s) d s \\
= & t e^{\frac{1}{2} \theta t^{2}} S_{t}^{H}-\theta t^{2} e^{\frac{1}{2} \theta t^{2}} \int_{0}^{t} s e^{\frac{1}{2} \theta s^{2}}\left(\int_{s}^{t} e^{-\frac{1}{2} \theta u^{2}} d u\right) d S_{s}^{H} \\
& +v t e^{\frac{1}{2} \theta t^{2}} \int_{0}^{t} e^{-\frac{1}{2} \theta s^{2}} d s \\
= & t e^{-\frac{1}{2} \theta t^{2}} S_{t}^{H}-\theta t e^{\frac{1}{2} \theta t^{2}} \int_{0}^{t} e^{-\frac{1}{2} \theta u^{2}}\left(\int_{0}^{u} s e^{\frac{1}{2} \theta s^{2}} d S_{s}^{H}\right) d u \\
& +v t e^{\frac{1}{2} \theta t^{2}} \int_{0}^{t} e^{-\frac{1}{2} \theta s^{2}} d s \\
= & t e^{\frac{1}{2} \theta t^{2}} S_{t}^{H}-\theta t e^{\frac{1}{2} \theta t^{2}} \int_{0}^{t} e^{-\frac{1}{2} \theta u^{2}} \xi_{u}^{H} d u \\
& +v t e^{\frac{1}{2} \theta t^{2}} \int_{0}^{t} e^{-\frac{1}{2} \theta s^{2}} d s .
\end{aligned}
$$

It follows from Lemma 4.1, Corollary 4.1, and Lemma 4.2 that

$$
\begin{gathered}
J_{0}^{H}(t ; \theta, \nu)-\left(\xi_{\infty}^{H}-\frac{\nu}{\theta}\right)=t e^{\frac{1}{2} \theta t^{2}} X_{t}^{H}-\left(\xi_{\infty}^{H}-\frac{\nu}{\theta}\right) \\
=t e^{\frac{1}{2} \theta t^{2}} S_{t}^{H}-\theta t e^{\frac{1}{2} \theta t^{2}} \int_{0}^{t} e^{-\frac{1}{2} \theta u^{2}}\left(\xi_{u}^{H}-\xi_{\infty}^{H}\right) d u \\
+\left(\xi_{\infty}^{H}-\frac{\nu}{\theta}\right)\left(-\theta t e^{\frac{1}{2} \theta t^{2}} \int_{0}^{t} e^{-\frac{1}{2} \theta u^{2}} d u-1\right) \rightarrow 0 \quad(t \rightarrow \infty)
\end{gathered}
$$

in $L^{2}$ and almost surely for all $\theta<0$ and $\frac{1}{2}<H<1$, as $t$ tends to infinity.

Theorem 4.2. Define the processes $J^{H}(n, \theta, v)=$ $\left\{J_{t}^{H}(n, \theta, v), t \geq 0\right\}, n \geq 1$ by

$$
\begin{aligned}
J_{n}^{H}(t ; \theta, \nu):=\theta t^{2}\left(J_{n-1}^{H}(t ; \theta, \nu)-(2 n-3) ! !\left(\xi_{\infty}^{H}-\frac{\nu}{\theta}\right)\right) & \\
n & =1,2, \ldots,
\end{aligned}
$$

for all $t \geq 0$, where $(-1) ! !=1$. Then, the convergence

$$
J_{n}^{H}(t ; \theta, \nu) \rightarrow(2 n-1) ! !\left(\xi_{\infty}^{H}-\frac{\nu}{\theta}\right)
$$

holds in $L^{2}$ and almost surely for every $n \geq 1$, as $t \rightarrow \infty$.

Proof. From the proof of Theorem 4.1, we find that the identities

$$
\begin{array}{r}
J_{0}^{H}(t ; \theta, \nu)-\left(\xi_{\infty}^{H}-\frac{\nu}{\theta}\right)=t e^{\frac{1}{2} \theta t^{2}} S_{t}^{H}+\theta t e^{\frac{1}{2} \theta t^{2}} \int_{0}^{t} e^{-\frac{1}{2} \theta u^{2}}\left(\xi_{u}^{H}-\xi_{\infty}^{H}\right) d u \\
+\left(\xi_{\infty}^{H}-\frac{\nu}{\theta}\right)\left(\theta t e^{\frac{1}{2} \theta t^{2}} \int_{0}^{t} e^{-\frac{1}{2} \theta u^{2}} d u-1\right) \\
J_{n}^{H}(t ; \theta, \nu)=\left(\xi_{\infty}^{H}-\frac{\nu}{\theta}\right) I_{n}(t, \theta)+t\left(\theta t^{2}\right)^{n} e^{-\frac{1}{2} \theta t^{2}} S_{t}^{H} \\
+\theta t\left(\theta t^{2}\right)^{n} e^{\frac{1}{2} \theta t^{2}} \int_{0}^{t} e^{\frac{1}{2} \theta u^{2}}\left(\xi_{u}^{H}-\xi_{\infty}^{H}\right) d u
\end{array}
$$

holds for all $t>0, n \geq 1$ and $\theta<0$, where $I_{n}(t, \theta)$ is given in Lemma 3.2. Thus, the theorem follows from Lemma 4.1, Corollary 4.1, Lemma 4.2 and Theorem 4.1 .

\section{SIMULATION}

We have applied our results to the following linear self-repelling diffusion driven by a sub-fBm $S^{H}$ with $\frac{1}{2}<H<1$ :

$$
d X_{t}^{H}=d S_{t}^{H}-\theta\left(\int_{0}^{t}\left(X_{t}^{H}-X_{s}^{H}\right) d s\right) d t+v d t, \quad X_{0}^{H}=0,
$$

where $\theta<0$ and $\nu \in \mathbb{R}$ are two parameters. We will simulate the process with $\nu=0$ in the following cases:

- $H=0.7$ and $\theta=-1, \theta=-10$, and $\theta=-100$, respectively (see, Figure 1, Figure 2, Figure 3, and Table 1, Table 2, Table 3);

- $H=0.5$ and $\theta=-1, \theta=-10$, and $\theta=-100$, respectively (see, Figure 4, Figure 5, Figure 6, and Table 4, Table 5, Table 6);

Remark 1. From the following numerical results, we can find that it is important to study the estimates of parameters $\theta$ and $\nu$.

\section{DATA AVAILABILITY STATEMENT}

The original contributions presented in the study are included in the article/Supplementary Material, further inquiries can be directed to the corresponding author.

\section{AUTHOR CONTRIBUTIONS}

All authors listed have made a substantial, direct, and intellectual contribution to the work and approved it for publication.

\section{FUNDING}

This study was funded by the National Natural Science Foundation of China (NSFC), grant no. 11971101. 


\section{REFERENCES}

1. Cranston M, Le Jan Y. Self Attracting Diffusions: Two Case Studies. Math Ann (1995) 303:87-93. doi:10.1007/bf01460980

2. Durrett RT, Rogers LCG. Asymptotic Behavior of Brownian Polymers. Probab Th Rel Fields (1992) 92:337-49. doi:10.1007/bf01300560

3. Pemantle R. Phase Transition in Reinforced Random Walk and RWRE on Trees. Ann Probab (1988) 16:1229-41. doi:10.1214/aop/1176991687

4. Benaïm M, Ledoux M, Raimond O. Self-interacting Diffusions. Probab Theor Relat Fields (2002) 122:1-41. doi:10.1007/s004400100161

5. Benaïm M, Ciotir I, Gauthier C-E. Self-repelling Diffusions via an Infinite Dimensional Approach. Stoch Pde: Anal Comp (2015) 3:506-30. doi:10.1007/ s40072-015-0059-5

6. Cranston M, Mountford TS. The strong Law of Large Numbers for a Brownian Polymer. Ann Probab (1996) 24:1300-23. doi:10.1214/aop/1065725183

7. Gauthier C-E. Self Attracting Diffusions on a Sphere and Application to a Periodic Case. Electron Commun Probab (2016) 21(No. 53):1-12. doi:10.1214/16-ecp4547

8. Herrmann S, Roynette B. Boundedness and Convergence of Some SelfAttracting Diffusions. Mathematische Annalen (2003) 325:81-96. doi:10.1007/s00208-002-0370-0

9. Herrmann S, Scheutzow M. Rate of Convergence of Some Self-Attracting Diffusions. Stochastic Process their Appl (2004) 111:41-55. doi:10.1016/ j.spa.2003.10.012

10. Mountford T, Tarrés P. An Asymptotic Result for Brownian Polymers. Ann Inst H Poincaré Probab Statist (2008) 44:29-46. doi:10.1214/07-aihp113

11. Shen L, Xia X, Yan L. Least Squares Estimation for the Linear Self-Repelling Diffusion Driven by $\alpha$-stable Motions. Appear Statist Probab Lett (2021) 181: 109259. doi:10.1016/j.spl.2021.109259

12. Sun X, Yan L. A Convergence on the Linear Self-Interacting Diffusion Driven by $\alpha$-stable Motion. Stochastics 93(2021):1186-1208. doi:10.1080/ 17442508.2020.1869239

13. Yan L, Sun Y, Lu Y. On the Linear Fractional Self-Attracting Diffusion. J Theor Probab (2008) 21:502-16. doi:10.1007/s10959-007-0113-y

14. Sun X, Yan L. The Laws of Large Numbers Associated with the Linear SelfAttracting Diffusion Driven by Fractional Brownian Motion and Applications. J Theoret Prob (2021). [Epub of Print]. doi:10.1007/s10959-021-01126-0

15. Gan Y, Yan L. Least Squares Estimation for the Linear Self-Repelling Diffusion Driven by Fractional Brownian Motion (In Chinese). Sci CHINA Math (2018) 48:1143-58. doi:10.1360/scm-2017-0387

16. Alós E, Mazet O, Nualart D. Stochastic Calculus with Respect to Gaussian Processes. Ann Prob (2001) 29:766-801. doi:10.1214/aop/1008956692

17. Bojdecki T, Gorostiza LG, Talarczyk A. Some Extensions of Fractional Brownian Motion and Sub-fractional Brownian Motion Related to Particle Systems. Elect Comm Probab (2007) 12:161-72. doi:10.1214/ecp.v12-1272

18. Bojdecki T, Gorostiza LG, Talarczyk A. Sub-fractional Brownian Motion and its Relation to Occupation Times. Stat Probab Lett (2004) 69:405-19. doi:10.1016/j.spl.2004.06.035

19. Bojdecki T, Gorostiza LG, Talarczyk A. Occupation Time Limits of Inhomogeneous Poisson Systems of Independent Particles. Stochastic Process their Appl (2008) 118:28-52. doi:10.1016/j.spa.2007.03.008

20. Bojdecki T, Gorostiza LG, Talarczyk A. Self-Similar Stable Processes Arising from High-Density Limits of Occupation Times of Particle Systems. Potential Anal (2008) 28:71-103. doi:10.1007/s11118-007-9067-z

21. Li M. Modified Multifractional Gaussian Noise and its Application. Phys Scr (2021) 96(1212):125002. doi:10.1088/1402-4896/ac1cf6

22. Li M. Generalized Fractional Gaussian Noise and its Application to Traffic Modeling. Physica A 579(202122):1236137. doi:10.1016/j.physa.2021.126138
23. Li M. Multi-fractional Generalized Cauchy Process and its Application to Teletraffic. Physica A: Stat Mech its Appl (2020) 550(14):123982. doi:10.1016/ j.physa.2019.123982

24. Li M. Fractal Time Series-A Tutorial Review. Math Probl Eng (2010) 2010:26, 2010. Article ID 157264. doi:10.1155/2010/157264

25. Shen G, Yan L. An Approximation of Subfractional Brownian Motion. Commun Stat - Theor Methods (2014) 43:1873-86. doi:10.1080/ 03610926.2013.769598

26. Shen G, Yan L. Estimators for the Drift of Subfractional Brownian Motion. Commun Stat - Theor Methods (2014) 43:1601-12. doi:10.1080/ 03610926.2012.697243

27. Sun X, Yan L. A central Limit Theorem Associated with Sub-fractional Brownian Motion and an Application (In Chinese). Sci Sin Math (2017) 47:1055-1076. doi:10.1360/scm-2016-0748

28. Tudor C. Some Properties of the Sub-fractional Brownian Motion. Stochastics (2007) 79:431-48. doi:10.1080/17442500601100331

29. Tudor C. Inner Product Spaces of Integrands Associated to Subfractional Brownian Motion. Stat Probab Lett (2008) 78:2201-9. doi:10.1016/ j.spl.2008.01.087

30. Tudor C. On the Wiener Integral with Respect to a Sub-fractional Brownian Motion on an Interval. J Math Anal Appl (2009) 351:456-68. doi:10.1016/ j.jmaa.2008.10.041

31. Tudor C. Some Aspects of Stochastic Calculus for the Sub-fractional Brownian Motion. Ann Univ Bucuresti Mathematica (2008) 24:199-230.

32. Ciprian A. Tudor, Analysis Of Variations For Self-Similar Processes. Heidelberg, New York: Springer (2013).

33. Yan L, He K, Chen C. The Generalized Bouleau-Yor Identity for a Subfractional Brownian Motion. Sci China Math (2013) 56:2089-116. doi:10.1007/ s11425-013-4604-2

34. Yan L, Shen G. On the Collision Local Time of Sub-fractional Brownian Motions. Stat Probab Lett (2010) 80:296-308. doi:10.1016/ j.spl.2009.11.003

35. Yan L, Shen G, He K. Itô's Formula for the Sub-fractional Brownian Motion Comm Stochastic Anal (2011) 5:135-59. doi:10.31390/cosa.5.1.09

36. Nualart D. Malliavin Calculus and Related Topics. 2nd ed. New York: Springer (2006).

37. Bertoin J. Sur une intégrale pour les processus á $\alpha$-variation borné. Ann Probab (1989) 17:1521-35. doi:10.1214/aop/1176991171

38. FöIllmer H, Calcul d'Itô Sans Probabilités, Azéme and Yor : Séinaire de Probabilités XV, Lecture Notes in Math, No 50. Springer-Verlag (1980). p. 143-5.

Conflict of Interest: The authors declare that the research was conducted in the absence of any commercial or financial relationships that could be construed as a potential conflict of interest.

Publisher's Note: All claims expressed in this article are solely those of the authors and do not necessarily represent those of their affiliated organizations, or those of the publisher, the editors and the reviewers. Any product that may be evaluated in this article, or claim that may be made by its manufacturer, is not guaranteed or endorsed by the publisher.

Copyright (C) 2022 Gao, Guo, Jin and Yan. This is an open-access article distributed under the terms of the Creative Commons Attribution License (CC BY). The use, distribution or reproduction in other forums is permitted, provided the original author(s) and the copyright owner(s) are credited and that the original publication in this journal is cited, in accordance with accepted academic practice. No use, distribution or reproduction is permitted which does not comply with these terms. 